\title{
Genome-wide analysis and transcriptomic profiling of the auxin biosynthesis, transport and signaling family genes in moso bamboo (Phyllostachys heterocycla)
}

Wenjia Wang ${ }^{1 \dagger}$, Lianfeng Gu ${ }^{1+}$, Shanwen Ye ${ }^{1}$, Hangxiao Zhang ${ }^{1}$, Changyang Cai ${ }^{1}$, Mengqi Xiang ${ }^{1}$, Yubang Gao', Qin Wang ${ }^{1}$, Chentao Lin ${ }^{1,2}$ and Qiang Zhu ${ }^{1 *}$ (D)

\begin{abstract}
Background: Auxin is essential for plant growth and development. Although substantial progress has been made in understanding auxin pathways in model plants such as Arabidopsis and rice, little is known in moso bamboo which is famous for its fast growth resulting from the rapid cell elongation and division.

Results: Here we showed that exogenous auxin has strong effects on crown and primary roots. Genes involved in auxin action, including 13 YUCCA (YUC) genes involved in auxin synthesis, 14 PIN-FORMED/PIN-like (PIN/PILS) and 7 AUXIN1/LIKE-AUX1 (AUX1/LAX) members involved in auxin transport, 10 auxin receptors (AFB) involved in auxin perception, 43 auxin/indole-3-aceticacid (AUX/IAA) genes, and 41 auxin response factors (ARF) involved in auxin signaling were identified through genome-wide analysis. Phylogenetic analysis of these genes from Arabidopsis, Oryza sativa and bamboo revealed that auxin biosynthesis, transport, and signaling pathways are conserved in these species. A comprehensive study of auxin-responsive genes using RNA sequencing technology was performed, and the results also supported that moso bamboo shared a conserved regulatory mechanism for the expression of auxin pathway genes; meanwhile it harbors its own specific properties.
\end{abstract}

Conclusions: In summary, we generated an overview of the auxin pathway in bamboo, which provides information for uncovering the precise roles of auxin pathway in this important species in the future.

Keywords: Moso bamboo, Auxin biosynthesis, Auxin transport, Auxin signaling, Gene expression

\section{Background}

Auxin acts as a central organization hub in controlling plant growth and development $[1,2]$. Auxin action can be achieved through different levels, mainly auxin concentration pathways including auxin biosynthesis and directional auxin transport, and auxin signaling pathways including auxin perception and signal transduction [3-5].

Although auxin biosynthesis is not fully understood in plants, genetic and biochemical studies have demonstrated

\footnotetext{
* Correspondence: zhuqiang@fafu.edu.cn

${ }^{\dagger}$ Equal contributors

'Basic Forestry and Proteomics Center (BFPC), Fujian Provincial Key Laboratory of Haixia Applied Plant Systems Biology, Haixia Institute of Science and Technology, Fujian Agriculture and Forestry University, Fujian 350002, China

Full list of author information is available at the end of the article
}

that the endogenous plant auxin indole-3-acetic acid (IAA) is mainly synthesized by a two-step reaction: $\operatorname{Trp}$ is first converted to indole-3-pyruvate (IPA) by TRYPTOPHAN AMINOTRANSFERASE OF ARABIDOPSIS (TAA) and then IAA is produced by YUC flavincontaining monooxygenase family proteins [6]. YUC proteins that encode flavin monooxygenases catalyze a rate-limiting step of the IPA pathway [7]. In Arabidopsis, 11 YUC family proteins act redundantly and cooperatively at various growth and developmental stages $[4,6]$.

IAA mainly exists in the protonated form (IAA-H) in the apoplast, whereas the deprotonated form (IAA-) becomes dominant inside the plant cell due to the $\mathrm{pH}$ changes [8]. The IAA-H form of auxin freely enters the cell via diffusion, and the transport of its anionic form 
(IAA ${ }^{-}$is mediated mainly by auxin influx transporters (AUX1/LAX proteins) and efflux transporters (ATP binding cassette B and PIN/PILS proteins) [5, 9]. In Arabidopsis, 4 amino acid permease-like family members (AtAUX1/ LAX1-3) regulate auxin uptake from the apoplast [10], whereas 8 PIN proteins (AtPIN1-PIN8) and 7 PILS members (AtPILS1-7) are responsible for the polar pump-off of auxin and determine the direction of auxin flow through tissues [5]. The AtPIN-like family of proteins (AtPILS) was identified based on predicted topological similarities with AtPIN proteins [11]. As AtPIN proteins, AtPILS proteins contain the so-called InterPro auxin carrier domain, which is predicted to have auxin transport function in silico. In Arabidopsis, AtPILS proteins mainly localize in the endoplasmic reticulum (ER), and regulate intracellular auxin accumulation and the rate of auxin conjugation [11, 12]. The proper concentration of auxin throughout the plant body is achieved by auxin biosynthesis and polar auxin transport.

Synthesized auxin is distributed to the site of its action in a directional manner $[5,9]$. The auxin regulatory module TIR1/AFB receptors-AUX/IAAs-ARFs, which is considered as the key components of the auxin signaling pathway in plant cells stimulates diverse auxin responses by coordinately controlling the expression of downstream genes [13]. In Arabidopsis, auxin binds to its receptor Transport Inhibitor Response 1 (TIR1), which belongs to a small gene family containing 5 additional members (AFB1-5). All of these 6 proteins redundantly function as nuclear auxin receptors [14-16]. TIR1/AFBs act as the specificity determiners for the SCF class of E3 ubiquitin ligases, which target substrate proteins for polyubiquitylation and subsequent degradation [17]. AUX/IAAs are negative regulators of the auxin signaling pathway [18]. Typically, AUX/IAA proteins have 4 conserved domains: domain I contains an ethylene responsive factor (ERF) associated amphiphilic repression motif and is required to recruit the transcriptional corepressor TOPLESS [19]; domain II is essential for auxin-induced AUX/IAA proteolysis [18]; and domains III and IV are involved in the homo- and hetero dimerization and interaction with the downstream auxin response factors (ARF) [20]. ARF proteins are a class of plant-specific B3type transcription factors which mediate auxin-dependent transcriptional regulation [21]. In Arabidopsis, 23 ARF proteins act as either activators or repressors of the downstream auxin-responsive genes by binding to the auxinresponsive cis-element (AuxRE: ['TGTCTC']) [21]. The auxin receptor-AUX/IAA-ARF module precisely and sensitively controls the response of plant cells to auxin: without auxin, AUX/IAA proteins negatively regulate the abundance of ARFs, and subsequently the expression of auxin responsive genes; whereas upon elevated auxin level, the auxin-dependent ubiquitination and degradation of Aux/IAA proteins mediated by $\mathrm{SCF}^{\mathrm{TIR} 1}$ (TIR1 perceives auxin) releases ARF proteins to activate the transcription of auxinresponsive genes [13, 22].

Moso bamboo is one of the most important nontimber forest products in the world, due to its great economic, cultural, and environmental value [23]. Moso bamboo is famous for its fast-growing culms which were controlled by cell division and cell elongation [24]. Although the mechanisms that control plant cell number and size are not fully understood, phytohormones, especially auxin have important roles in the regulation and coordination of plant cell proliferation and elongation [25]. However, to our knowledge, no systematic study of the auxin pathway has been reported in moso bamboo. The genomic sequence of moso bamboo was recently released [26], providing an excellent opportunity to perform a comprehensive genome-wide analysis of the gene families related to auxin action. Here, a genome-wide search was carried out to identify auxin action-related genes in moso bamboo. A total of $13 Y U C$ genes involved in auxin biosynthesis, 13 PIN/PILS and 7 AUX1/ $L A X$ family members involved in auxin transport, $10 \mathrm{pu}-$ tative auxin receptors involved in auxin perception, and $43 A U X / I A A s$ and $41 A R F$ s involved in auxin signaling were identified from the moso bamboo genome. Next we analyzed the phylogenetic relationships of auxin pathway orthologs from moso bamboo, Arabidopsis, and rice. Moreover, to generate a general overview of the auxin-response transcriptome in moso bamboo, we treated the plants with exogenous auxin and performed RNA-Sequencing (RNA-Seq) analyses. Our data provide a foundation for further investigations of the role of auxin in regulating moso bamboo development at the genetic and biotechnological levels.

\section{Methods \\ Isolation of gene families related to auxin action in moso bamboo}

The gene annotations and genomic sequences of moso bamboo were downloaded from the bamboo genome database (BambooGDB, http://www.bamboogdb.org/). To identify the members involved in auxin pathway in moso bamboo, we retrieved proteins of 11 AtYUCs, 8 AtPINs, 6 AtPILSs, 4 AtAUX1/LAXs, 6 AtTIR1/AFBs, 29 AtAUX/IAAs and 23 AtARFs from the Arabidopsis genome database, and used as query sequences against the moso bamboo database using basic local alignment search tool (BLAST) search. All sequences with an e-value $\leq 10^{-11}$ and a score value $\geq 100$ were used as new queries for the 2nd cycle's search to avoid missing additional orthologs. In addition, the derived sequences were used for further protein domain analysis using the Pfam program (http:// 
pfam.xfam.org) and SMART (http://smart.embl-heidelberg.de/). To exclude the duplicated genes, all the candidates were aligned using the ClustalW program [27]. Information about the coding sequences, full-length sequences, and amino sequences were obtained from the bamboo genome database using the BLAST program.

\section{Gene structure, conserved motif, and protein information analyses}

The genomic and cDNA sequences of each predicted gene were downloaded from the moso bamboo genome database, and their intron distribution patterns and intro-exon boundaries were analyzed using JBrowse software as previously described [28]. The conserved motif was derived using the NCBI conserved domain search (https://www.ncbi.nlm.nih.gov/Structure/cdd/wrpsb.cgi) or the online tool Pfam. The protein sequence was analyzed using DNAman software.

\section{Phylogenetic tree building and prediction of amino acid composition}

Multiple sequence alignments of the auxin actionrelated proteins (YUC family, PIN/PILS family, AUX1/ LAX family, AFB family, AUX/IAA family and ARF family) were performed using the ClustalW program with the default parameters [27]. The results were visualized using Editplus (https://www.editplus.com/). The phylogenetic tree was constructed from the protein sequences given above using MEGA6 program (http://www.megasoftware.net/mega.php) with the neighbor-joining (NJ) method, and the boot strap analysis was performed using 1000 replicates as described previously [29].

\section{Plant materials and auxin treatment}

To determine the effects of auxin on the growth of moso bamboo, we germinated and grew bamboo seeds in the soil in a greenhouse at $26{ }^{\circ} \mathrm{C}$ with the photoperiod of $16 \mathrm{~h}$ light $/ 8 \mathrm{~h}$ dark for 1 month. Various concentrations of naphthaleneacetic acid (NAA, $100 \mathrm{nM}, 500 \mathrm{nM}$, and $5 \mu \mathrm{M})$ were sprayed the above-ground parts and watered the roots of the seedlings for 2 weeks at 2-day intervals, and pictures were taken for further statistical analysis using Image J software. At least 3 independent biological repeats were performed.

For materials used in RNA-Seq analysis, seeds were sterilized with chlorine gas for 3-5 h and were then put on Murashige and skoog (MS) agar to germinate and grow vertically. 1-month-old seedlings were sprayed with $5 \mu \mathrm{M}$ NAA, $5 \mu \mathrm{M}$ IAA or $5 \mu \mathrm{M}$ Indole-3-butytric acid (IBA) for $4 \mathrm{~h}$ at 1 -h intervals, and the root parts were dissected for RNA extraction. For the time course experiments, 1-month-old seedlings were sprayed with $5 \mu \mathrm{M}$ NAA, and the roots were harvested at different time points $(0,2,4,6,8$ and $12 \mathrm{~h})$ for further qPCR analysis.

\section{RNA extraction and RNA-Seq analysis}

Total RNA from moso bamboo roots were extracted using the RNeasy Mini Kit (QIAGEN, China) according to the manufacturer's instructions. $10 \mu \mathrm{g}$ total RNA were used for RNA-Seq analysis using the Illumina Hiseq2500 Sequencer platform (BerryGenomics, China) with paired-end sequencing, and 3 independent biological repeats were performed. In total, 6 strand-specific RNASeq libraries were sequenced in this study using the deoxyuridine triphosphate (dUTP) method [30]. The paired-end reads were aligned to the moso bamboo genome using tophat-2.0.11 with anchor length more than 8 nt for spliced alignments [31]. Only reads that could be uniquely aligned were retained for subsequent analysis. The expression levels of each gene were normalized as fragments per kilobase of transcript per million mapped reads (FPKM) [32]. The $p$-value and false discovery rate (FDR) were calculated using the edgeR package developed in Bioconductor [33]. A fold change in the expression $>1.5$ and an FDR $<0.01$ were considered to be the threshold for differentially expressed genes (DEGs). To verify the RNA-Seq results, qPCR was used as previously described [34]. The primers used for qPCR are listed in the Additional file 1: Table S7. We used DAVID [35] to test the statistical enrichment of the differentially expressed genes in the KEGG pathways.

\section{Results}

\section{Effects of exogenous auxin on moso bamboo}

Auxin controls nearly every aspect of plant growth and development, but until now no reports showed its role in bamboo growth. To test its effects on the growth of bamboo seedlings, we treated the 1-month-old seedlings with various concentration of synthetic auxin NAA for another 2 weeks. Our results showed that bamboo root is sensitive to the exogenous NAA treatment, and NAA affects the root architecture in a dose-dependent manner, low concentration (100 nM and $500 \mathrm{nM}$ ) of NAA promoted the formation and growth of crown roots, while an inhibition of primary root and lateral root growth was observed at a higher concentration of auxin $(5 \mu \mathrm{M})$ (Fig. 1a and b). Under our experimental conditions, we did not find significant differences in the above-ground plant architecture under our experimental conditions (Fig. 1a and b).

\section{Identification of gene families related to auxin action in moso bamboo}

Extensive evidences showed that the auxin plays its function through controlling its homeostasis, transport and signaling (Additional file 2: Figure S1) [13, 36, 37]. As a first step to reveal the role of auxin in affecting bamboo root growth, we performed a genome-wide analysis to identify the auxin related genes in moso bamboo, 


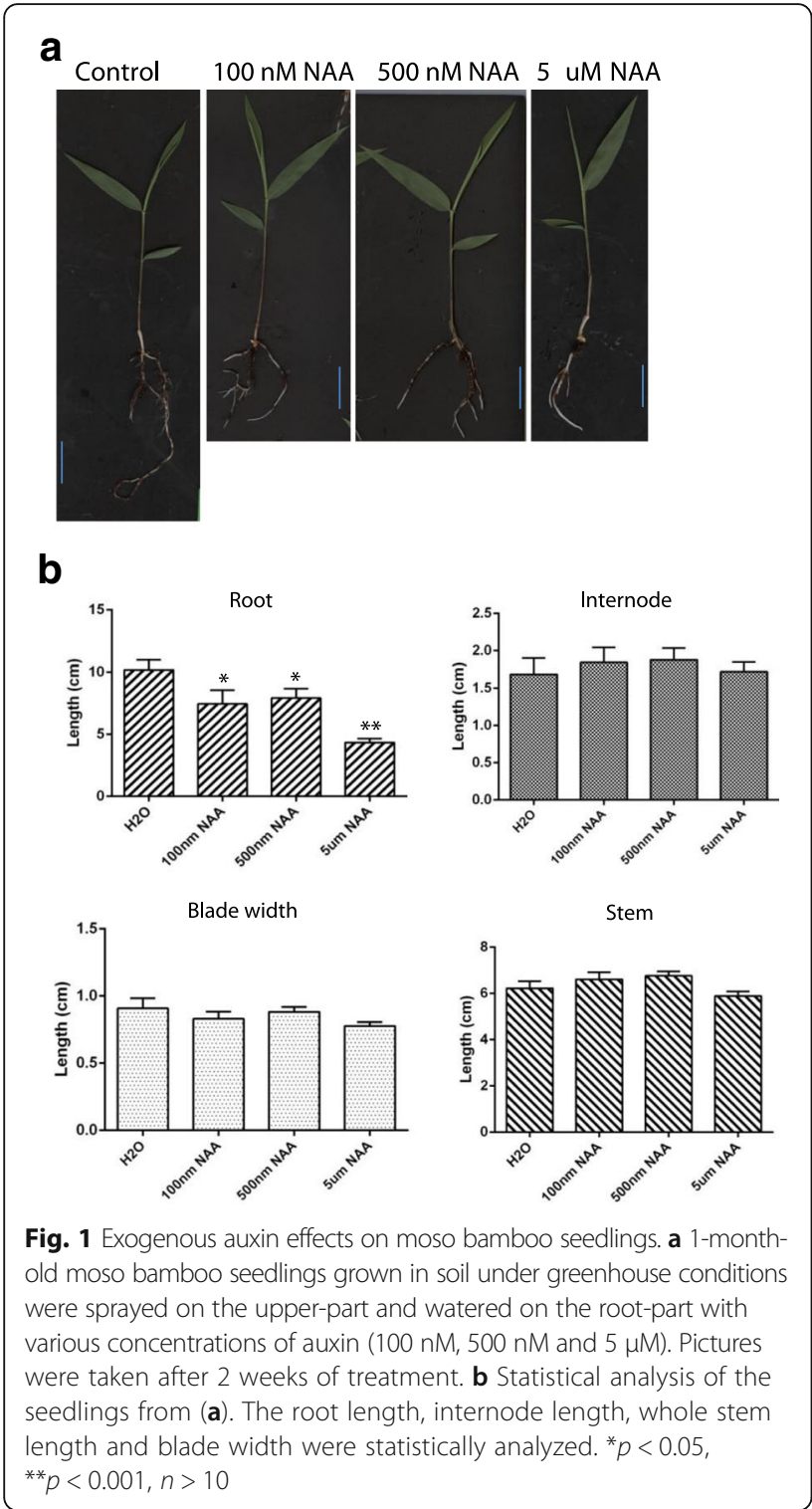

including $Y U C$ family genes for auxin biosynthesis, PIN/ PILS and AUX1/LAX families for polar auxin transport, $A F B$ family for auxin perception, and $A U X / I A A$ and $A R F$ families for auxin signaling.

\section{Auxin biosynthesis}

Plant cells exhibit concentration-dependent auxin responses by tightly controlling cellular auxin levels [38]. Local auxin biosynthesis regulated by $Y U C$ family genes plays key roles in auxin accumulation $[5,9]$.

To identify YUC genes in moso bamboo, we searched the moso bamboo genome database using the Arabidopsis YUC proteins sequences as the queries. Combined with conserved domain analysis using Pfam or SMART, $13 Y U C$ genes were identified in moso bamboo genome, and the phylogenetic analysis of predicted full-length YUC protein sequences were performed using neighbor-joining method (Fig. 2a). Our further search in the moso bambooGDB with these 13 YUCs did not identify additional $Y U C$ genes. We named these genes PhYUC1-PhYUC13 based on the scaffold number (Fig. 2a, Additional file 1: Table S1). Analyses of the protein domains using the Pfam program showed that all of these proteins contained the conserved flavin-binding monooxygenase-like domain as reported in other species [39], whereas some PhYUC proteins have their own specific structures. For example, all PhYUC proteins except PhYUC3 contain FAD-binding motif ['GxGxxG']. PhYUC1, 4, 9 and 11 have the classical ATG-containing motif 1 ['Y(x)7ATGEN(x)5P'], while PhYUC5, 6, 7, 8, 10, 12, and 13 share a conserved motif $\left[{ }^{\prime} \mathrm{D}(\mathrm{x}) 4 \mathrm{CI} / \mathrm{NG}(\mathrm{x}) 5 \mathrm{P}\right]$ in this region. The FMO identifying motif ['FxGxxxHxxxY/F'], NADPH-binding motif ['GxGxxG'], and ATG-containing motif 2 ['(F/L) ATGY'] are conserved in all PhYUC proteins except PhYUC1 and PhYUC2 (Additional file 2: Figure S2A). These structural differences suggest the possible functional diversity among the PhYUC proteins.

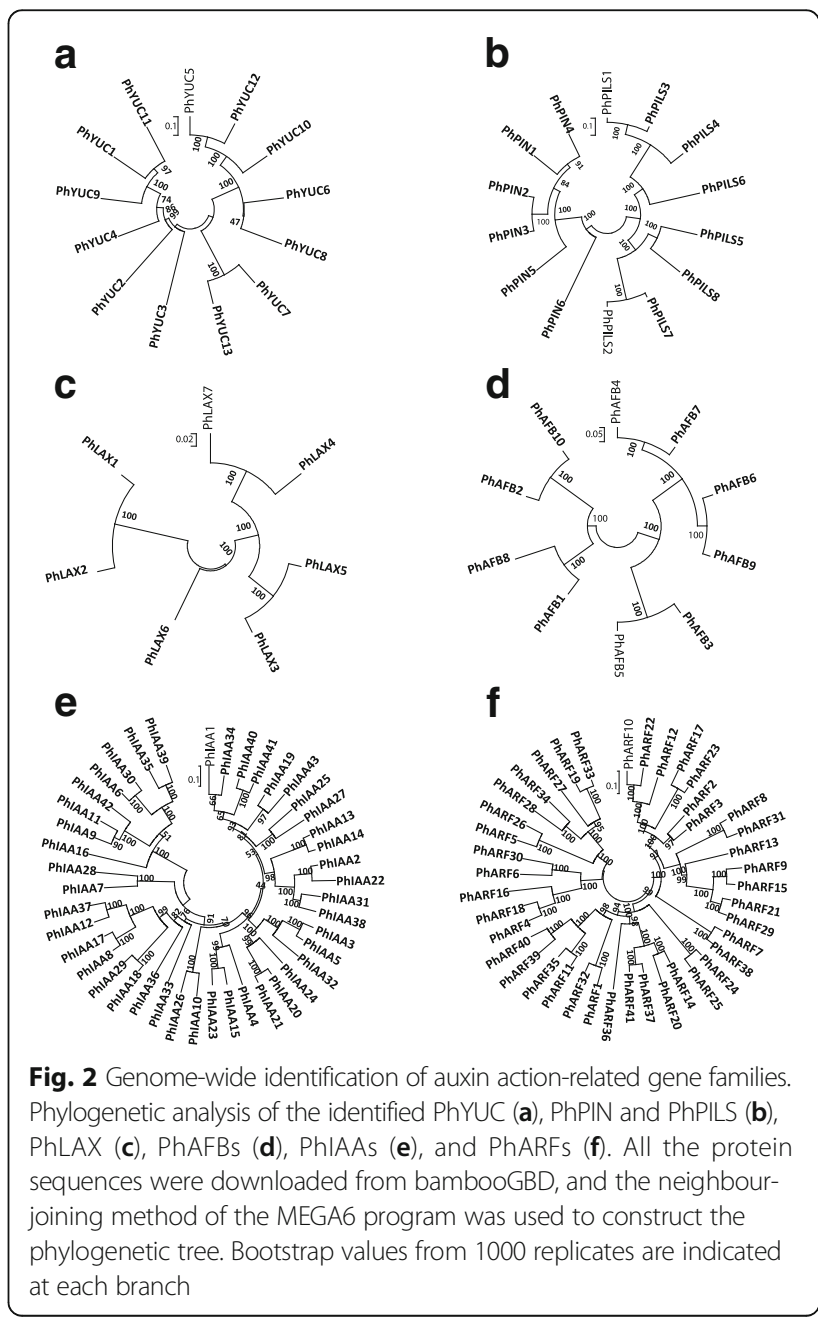


The sizes of the deduced PhYUC proteins varied markedly. The open reading frame (ORF) lengths of the PhYUC genes range from $258 \mathrm{bp}$ (PhYUC11) to $1656 \mathrm{bp}$ (PhYUC12) with the predicted molecular masses varied from 9.4 to $170.6 \mathrm{kDa}$ (Additional file 1: Table S1). To understand the gene structures of the PhYUC genes, the organization of exons and introns for each gene was obtained by comparing the cDNA sequences with the corresponding genomic sequences. The results showed that all members of the PhYUC family contained introns, ranging from 2 to 6 in numbers (Additional file 2: Figure S2B). The detailed information of the PhYUCs including the number of exons and introns, open reading frame lengths, translation lengths, molecular weights, conserved domains, and their putative subcellular localizations were listed in the Additional file 1: Table S1.

\section{Auxin transport}

After synthesis, auxin establishes the auxin gradient at the site for its function through auxin transport, a process which is controlled mainly by auxin efflux transporter PIN/PILS and influx transporter AUX1/ LAX [5, 9]. To identify the PIN and $A U X 1 / L A X$ members in moso bamboo, we used AtPIN/PILS and AtAUX1/LAX proteins from Arabidopsis as BLASTP queries to search for the moso bamboo orthologs in the bambooGDB. The Hidden Markov model (HMM) profiles (Pfam 01490: transmembrane amino acid transporter protein; Pfam 03547: membrane transport protein) were also employed to identify the PhLAX, PhPIN, and PhPILS protein families. A total of 6 PhPIN genes, 8 PhPILS and 7 PhLAX genes were identified and we further performed the phylogenetic analysis of their predicted full-length protein sequences using neighbor-joining method (Fig. 2b and c). All the PIN genes have similar intron/exon distribution patterns, and most of them have a large first exon that encodes the N-terminal transmembrane segments [5]. PhPIN genes contain 5-7 exons (Additional file 2: Figure S2C), and similar conserved exon/intron organization patterns have also been found in other plant species $[40,41]$. As the case in Arabidopsis, PIN transporters in moso bamboo were classified into long PINs and short PINs based on the length of predicted proteins. The typical long PINs contained 547-611 aa (PhPIN1-PhPIN4), while the short PINs contain only 332 aa or 470 aa (PhPIN6 and PhPIN5, respectively) (Additional file 1: Table S2). Multiple sequence alignment results showed that the sequences of the $\mathrm{N}$ - and $\mathrm{C}$-terminal transmembrane regions were highly conserved (Additional file 2: Figure S2D).

The ORFs of PhPILS genes range from $1023 \mathrm{bp}$ (PhPILS7) to 1296 bp (PhPILS1) (Additional file 1: Table S2). The gene structures of the PhPILS genes showed that their exon/intron numbers were different: PhPILS1,
PhPILS3, and PhPILS4 contained 10 exons, PhPILS2 and PhPILS7 contained 9 exons, and PhPILS5 and PhPILS8 contained 8 exons (Additional file 2: Figure S2E). Interestingly, PhPILS6 contained only 1 exon (Additional file 2: Figure S2E), similarly to the ZmPILS5 gene in maize [40]. As PhPIN proteins, multiple sequence alignment confirmed the high similarity among the PhPILS proteins. The results also revealed that the sequences of the $\mathrm{N}$ - and $\mathrm{C}$ - terminal transmembrane regions were highly conserved while the central hydrophilic region was variable (Additional file 2: Figure S2F), indicating the functional similarity among the members of the PhPILS family.

AUX/LAX proteins are required to establish the auxin gradient by mediating auxin influx transport [42]. In total, 7 auxin influx transporters (PhLAX1PhLAX7) were identified in moso bamboo genome (Fig. 2c). The exon numbers of PhLAX ranged from 6 (PhLAX6) to 8 (PhLAX4 and PhLAX7) (Additional file 2: Figure S2G), and the ORF lengths varied from $1431 \mathrm{bp}$ (PhLAX6) to $1593 \mathrm{bp}$ (PhLAX3) (Additional file 1: Table S3). Based on the multiple sequence alignment results, all members of this family shared conserved sequences (Additional file 2: Figure $\mathrm{S} 2 \mathrm{H})$. Moreover, all of these proteins were predicted to localize to the cell membrane (Additional file 1: Table S3), indicating that they have similar functions. The detailed information of these auxin transportrelated genes is listed in Additional file 1: Tables S2 and S3.

\section{Auxin signaling}

In plants, the auxin receptor-AUX/IAA-ARF module consists of the key components involved in auxin signaling [3]. In moso bamboo, 10 putative auxin receptors that showed highly similar protein sequences were identified, and the phylogenetic tree of their predicted full-length protein sequences was generated using neighbor-joining method (Fig. 2d). The exon number of these genes ranged from 3 to 4 (Additional file 2: Figure $\mathrm{S} 3 \mathrm{~A})$, the ORFs varied from $1632 \mathrm{bp}$ (PhAFB5) to 1881 bp (PhAFB6) (Additional file 1: Table S4).

AUX/IAA proteins are the direct targets of auxin receptors [14, 43]. 43 AUX/IAA proteins were identified in moso bamboo genome, and the corresponding phylogenetic tree was generated using neighbor-joining method (Fig. 2e). The ORF lengths ranged from $252 \mathrm{bp}$ (PhIAA19) to 2523 bp (PhIAA30) (Additional file 1: Table S5). The number of exons ranged from 2 to 18 (Additional file 2: Figure S3C). Sequence alignment analysis showed that most of the PhIAA proteins had typical domains I-IV. However, the diversity in the conserved domains was also observed (Additional file 2: Figure S3D). For example, the classical domain I ['TELRLGLPG'] was 
found in PhIAA1, 34, 43, 40, 41, 25, and 27, while a similar domain ['LR/K/T/ELXLXXPG'] was found in PhIAA2, 4, 8, 12-15, 17-18, 20-24, 29, 31, and 36-38. Moreover, in this region, a conserved domain ['MRFK/RMR/ KFRFEG'] was found in PhIAA6, 9, 11, 30, 35, 39, and 42. Whether these three domains have similar functions to that of the typical domain I needs to be further investigated (Additional file 2: Figure S3D). The other PhIAAs either lacked the domain I (PhIAA19 and PhIAA3) or had a poorly conserved domain I (PhIAA3, 5, 7, 10, 26, 28, and 32). Domain II ['VGWPP'] was found in most of the PhIAAs, however, some members did not contain domain II (PhIAA19 and PhIAA34) or had poorly conserved domain II sequences. Moreover, we found a novel conserved domain in this region ['LFGIXL'] with unknown function (Additional file 2: Figure S3D). Some members lacked either domain III (PhIAA19 and PhIAA22) or domain IV (PhIAA1, 34, and 28) (Additional file 2: Figure S3D).

Once the AUX/IAA protein was degraded, the ARF activities were released to activate downstream genes by binding to the AuxRE cis-elements ['TGTCTG'] in their promoters. A total of $41 A R F$ members were identified in the bamboo genome, and the phylogenetic tree was built with the sequences of their proteins using neighbor-joining method (Fig. 2f). The ORF lengths of the PhARFs ranged from $1284 \mathrm{bp}$ (PhARF3) to $3774 \mathrm{bp}$ (PhARF10) (Additional file 1: Table S6). To understand the structural components of the PhARF genes, the exon and intron organizations of the genes were determined by comparing the cDNA sequences with the corresponding genomic DNA sequences. The coding sequences of all family members contain introns, and the number of exons ranged from 2 (PhARF33) to 17 (PhARF1) (Additional file 2: Figure S3E). To explore the structural diversity and predict the functions of ARFs in moso bamboo, a motif analysis was performed using the NCBI conserved domain search engine (https:// www.ncbi.nlm.nih.gov/Structure/cdd/wrpsb.cgi). The results showed that all 41 putative PhARFs contained the highly conserved B3 DNA binding domain (DBD) (Additional file 2: Figure S3F and G). In Arabidopsis, the AtARF3, 13 and 17 proteins do not contain the carboxy-terminal dimerization domain (CTD domain) that is involved in the protein-protein interaction by dimerizing with AUX/IAA or with ARFs [21, 22]. Similarly to those proteins from Arabidopsis, the 12 ARF proteins in moso bamboo (PhARF2, 4, 6, 16, 18, 19, 25, $26,28,30,33,34$, and 36) also lacked this conserved domain (Additional file 2: Figure S3F and G). In summary, the moso bamboo ARF proteins have the typical B3 DBD domains that were required for binding to the AuxRE cis- elements, while their structural variations implied that the moso bamboo genome changed significantly during its evolutionary history, indicating the functional diversities of these ARF proteins.

\section{Phylogenetic analysis of the gene families related to auxin action}

To explore the possible roles of auxin action-related genes in moso bamboo and to understand their phylogenetic relationships, we constructed a phylogenetic tree using the proteins from rice, Arabidopsis, and moso bamboo. In general, the auxin action-related genes from bamboo showed high similarity to their orthologs from Arabidopsis and rice (Additional file 2: Figure S4).

The 38 members of YUC families in Arabidopsis, rice, and bamboo could be grouped into 3 classes. Of these proteins, PhYUC1, PhYUC9 and PhYUC11 belonged to class I (Additional file 2: Figure S4A) and were homologous to the genes OsYUC5, OsYUC7, and OsYUC1 respectively. PhYUC2 and PhYUC4 belonged to class II, and the PhYUC4 gene has two orthologs in rice OsYUC9 and OsYUC10. In rice, OsYUC9, OsYUC10, and OsYUC11 are highly expressed in the developing grains, and these genes are important for increases in the levels of IAA during grain development [44]. The highly close phylogenetic relationships of these genes indicated their potential function in bamboo grain development. Notably, the PhYUCs belonging to class III were not closely related to any rice or Arabidopsis YUC genes (Additional file 2: Figure S4A). This result probably reflects a diverging trend during the evolution of the YUC family members across different plant species.

Of the auxin receptor-like genes, the numbers of auxin receptor family in moso bamboo (10 members) was slightly expanded compared with that in Arabidopsis (6 members) or rice ( 8 members), and most of these genes are high closely related to the AFBs from these other two species (Additional file 2: Figure $\mathrm{S} 4 \mathrm{~B})$. All the AFBs members can be grouped into 3 classes, and all AFBs from Arabidopsis and bamboo are grouped into class I and class II (Additional file 2: Figure S4B). Based on the phylogenetic tree analysis, PhAFB10 and PhAFB2 are closed to the Arabidopsis TIR1, which is well-characterized as the firstidentified auxin receptor. It will be very interesting to determine the functions of these two genes in moso bamboo.

Although the PIN and PILS proteins are closely related, they belong to two separate groups in moso bamboo as in Arabidopsis and other species [11]. All PIN and PILS genes could be further divided into 3 subclasses (I-III) (Additional file 2: Figure S4C). In the PILS family, three pairs of PILS orthologs were identified between bamboo and rice: PhPILS8/ OsPILS7a, PhPILS6/OsPILS2, and PhPILS4/OsPILS6 
(Additional file 2: Figure S4C). In the PIN family, PhPIN1 belonged to the second group and was phylogenetically close to OsPIN10a and OsPIN10b, which are monocotspecific and have a long central hydrophilic domain. Based on the expression patterns of these genes in rice, they are involved in tillering [41, 45]. PhPIN2 and PhPIN3 are closely related to OsPIN1a, which is crucial for the negative phototropic curvature of the rice root [46]. PhPIN4 is the orthologs of the OsPIN1c and OsPIN1d from rice and AtPIN1 from Arabidopsis, which have broad effects on plant development [45], indicating the important role of PhPIN4 in moso bamboo development. Interestingly, compared with the PIN subfamily, PhPIN6 is more closely related to the PILS subfamily (Additional file 2: Figure S4C).

The AUX1/LAX proteins could be divided into 2 major classes: PhLAX3, 4, 5, 6, and 7 belonged to class I, while PhLAX1 and 2 belonged to class II (Additional file 2: Figure S4D). PhLAX3 and PhLAX5 are closely related to OsLAX2, whereas PhLAX4 and $P h L A X 7$ are closely related to OsLAX4; the PhLAX1 and PhLAX2 genes were closely related to OsLAX1 (Additional file 2: Figure S4D). These results may reflect the occurrence of a whole-genome duplication event from moso bamboo and rice [26].

The AUX/IAA family is significantly expanded in moso bamboo (43 members) compared with those of Arabidopsis $(29$ members) and rice (24 members). As reported in other species [47, 48], AUX/IAA proteins in moso bamboo can be divided into 6 major classes (I-VI). Group I consisted of 12 PhIAA proteins, 6 OsIAA proteins, and 10 AtIAA proteins that form 12 sister pairs (4 PhIAAOSIAA pairs, 4 PhIAA-PhIAA pairs, 4 AtIAA-AtIAA pairs). Group II contained 9 PhIAA proteins, 7 OsIAA proteins and 5 AtIAA proteins, which structure 7 sister pairs. Group III-VI contained 12 combined sister pairs. In total, 31 sister pairs were identified, and interestingly, no PhIAA-AtIAA pair was found (Additional file 2: Figure S4E). PhIAA15 is closely related to OsIAA1 in rice, and the overexpression of OsIAA1 effectively inhibits root elongation and shoot growth [49]. PhIAA13 and PhIAA14 are phylogenetically close to OsIAA11, the overexpression of which leads to the loss of lateral roots in rice [50].

In regards to AUX/IAAs, the moso bamboo genome contains much more PhARF members (41 members) compared to the genomes of Arabidopsis (23 members) and rice (25 members) (Additional file 2: Figure S4F). The phylogenetic distribution results showed that the ARF genes from these three species could be divided into 5 major classes (I-V). Thirty-one members were clustered into class I (12 members from moso bamboo), 21 members were clustered into class II $(9$ members from moso bamboo), and 11 members (4 PhARF) and 15 members (7 PhARF) were clustered into classes III and IV respectively. Only two members from Arabidopsis (AtARF2) and rice (OsARF20) were classified into class V (Additional file 2: Figure S4F).

\section{Auxin treatment induces broad changes in transcriptional activity}

Under our experimental conditions, the roots of moso bamboo were sensitive to exogenous NAA treatment (Fig. 1). Our next step was to check the global gene expression changes in response to exogenous auxin by RNA-Seq. To optimize the conditions for this experiment, first we treated the bamboo seedlings with $5 \mu \mathrm{M}$ NAA and the roots were harvested at different time points $(0,2,4,6,8$, and $12 \mathrm{~h}$ ) for testing the expression patterns of selected auxin responsive genes. The genes we checked were:Ph01003158G0110 and Ph01000099G0730 for Gretchen Hagen3 (GH3 family); Ph01000788G0760 for the lateral organ boundaries domain (LBD family); Ph01004534G0130 for small auxin-up RNA (SAUR family); Ph01000025G1600, Ph01000554G0550, Ph01000592G0620 and Ph01000075G0200 for AUX/IAA family, whose orthologs in model plants such as Arabidopsis and rice were used as the markers for plant response to exogenous auxin treatment [51]. Our results showed that $5 \mu \mathrm{M}$ NAA effectively changed the expression of these marker genes, and in most cases 4 h' treatment had the strongest effects (Additional file 2: Figure S5). Therefore we treated bamboo seedlings with $5 \mu \mathrm{M}$ NAA for $4 \mathrm{~h}$ and harvested the roots for RNA-Seq analysis, with the DMSO treated roots as the control. In general, with a cutoff of $P<0.05$ and a fold change $>1.5$, we identified 991 down-regulated genes and 1288 up-regulated genes in the root $4 \mathrm{~h}$ after treatment with $5 \mu \mathrm{M}$ NAA (Fig. 3, Additional file 3: Table S8). Our results from the Pearson correlation analysis showed that the correlations among all samples were quite high $(>0.95)$, and the biological replicates could cluster well together (Additional file 2: Figure S6). To determine the reliability of the RNA-Seq results, we selected 28 differentially expressed genes related to auxin action and performed quantitative real time PCR (qRT-PCR). The results indicated a close correlation between RNA-Seq and qRT-PCR data (Additional file 2: Figure S7).

The upregulated- and downregulated-genes were classified into many functional categories, including stress response, developmental processes, cell organization and biogenesis, signal transduction, and other processes. Moreover, a significant number of the upregulated and downregulated genes were distributed across many cellular components, such as cell wall, membrane and chloroplast, which have a broad molecular function (Fig. 3). These results suggested that auxin stimulates broad transcriptional changes in the root of moso bamboo as in other species. 


\section{a}

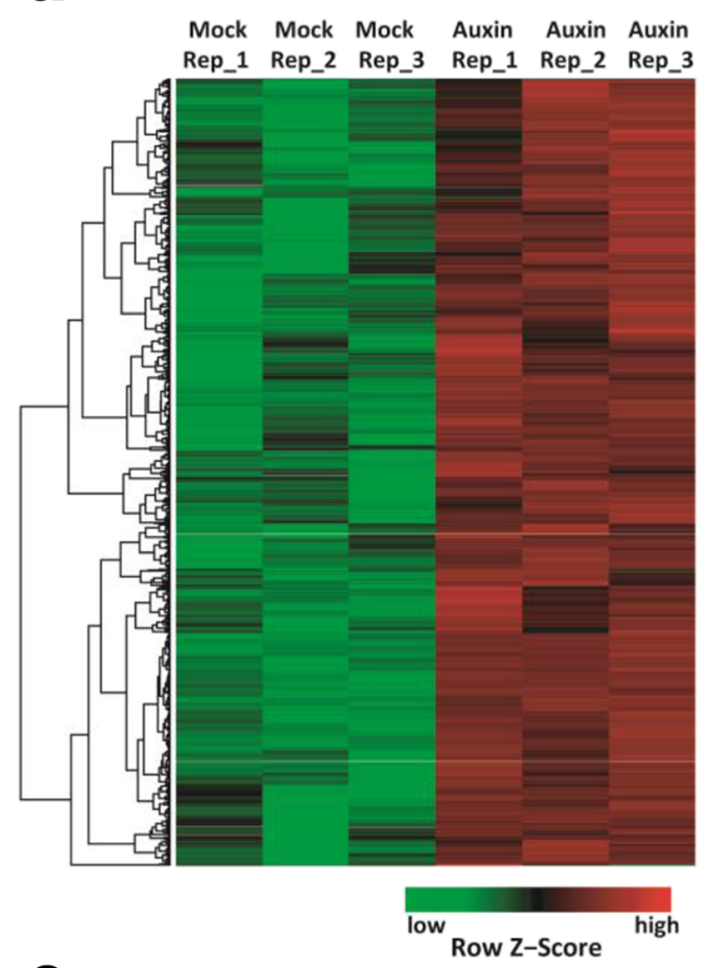

C

Mock Mock Mock Auxin Auxin Auxin Rep_1 Rep_2 Rep_3 Rep_1 Rep_2 Rep_3

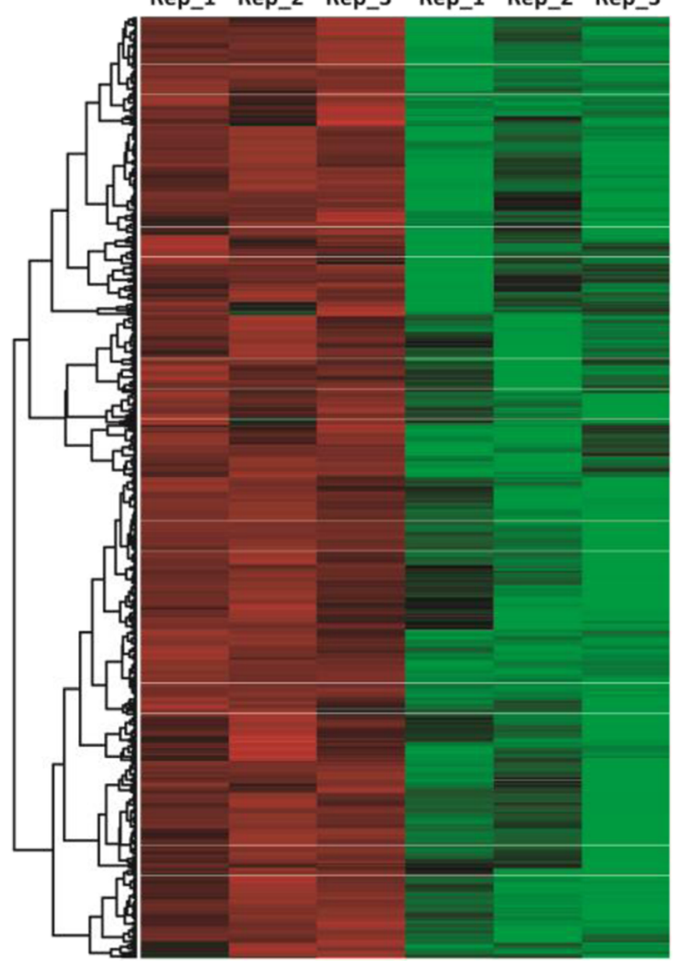

low Row Z-Score high b Functional Categorization by loci for: GO Biological Process $\mathrm{N}=499$

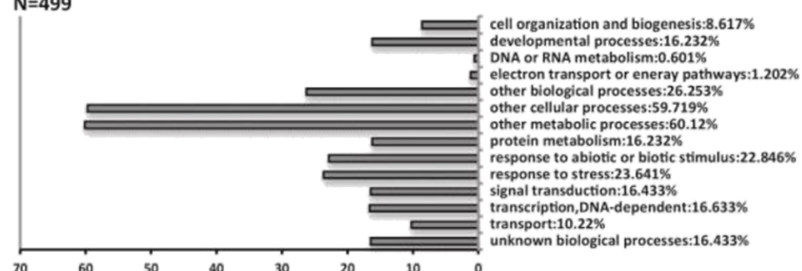

Functional Categorization by loci for: GO Cellular Component $\mathrm{N}=547$

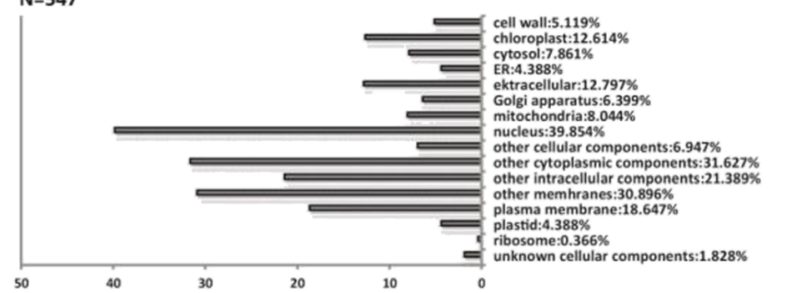

Functional Categorization by loci for: GO Molecular Function $\mathrm{N}=516$
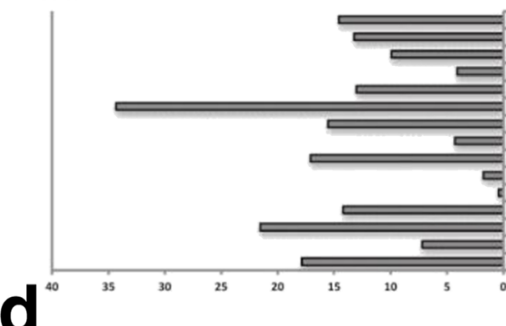

NA or RNA binding: $14.535 \%$ kydrolase activity:13.178 nucleic acid binding:4.07\% nucleotide binding:12.984\% ther binding: $34.302 \%$

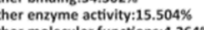
etein binding: $17.054 \%$ receptor binding or activity: $1.744 \%$ structural molecule activity:0.388\% ransferase activity:21.512\%

d

Functional Categorization by loci for:GO Biological Process $\mathrm{N}=417$

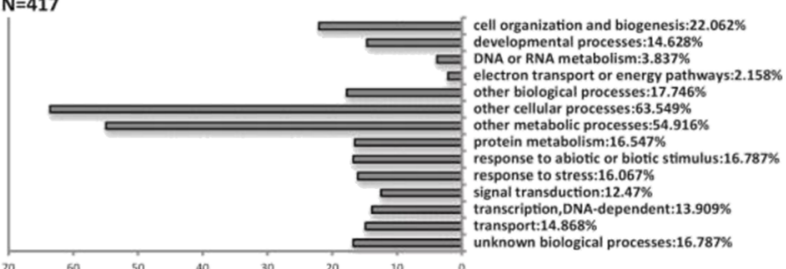

${ }^{70}{ }^{60}{ }^{50}{ }^{50}{ }^{40}{ }^{30} \stackrel{20}{20}^{10}{ }^{\circ}{ }^{\circ}$

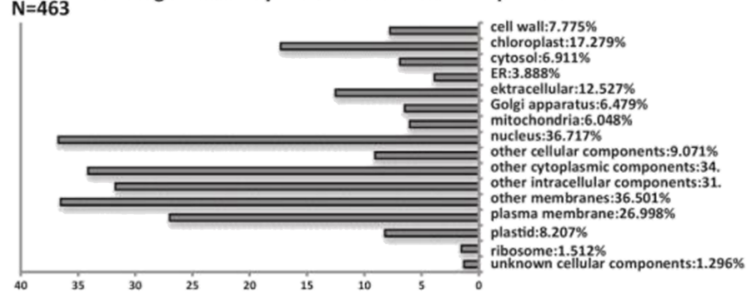

Functional Categorization by loci for: GO Molecular Function $\mathrm{N}=431$

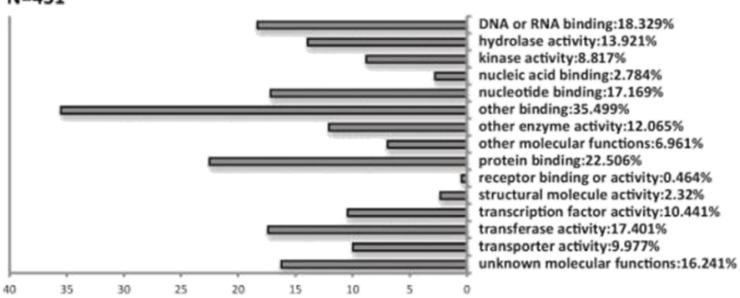


(See figure on previous page.)Fig. 3 Overview of the auxin response genes in moso bamboo. Six samples from auxin treated and mock treated moso bamboo roots collected and were subjected to RNA-Seq analysis. Heatmap representation and hierarchical clustering of the differentially expressed transcript clusters for the up-regulated (a) or down-regulated (c) in response to auxin treatment are shown. Numbers on the nodes display major gene groups based on expression patterns. Color scale shows log2 signal intensity values. GO enrichment analyses for up-regulated genes (b) and down-regulated genes (d) in response to auxin. Best hits were aligned to the GO database, and most consensus sequences were grouped into three major functional categories: biological process, cellular component, and molecular function. Red indicates up-regulated genes; green indicates down-regulated genes

\section{Expression changes of genes related to auxin action in response to treatment with exogenous auxin}

We further examined the auxin response of auxin biosynthesis, transport, and signaling pathway genes from RNASeq datasets, and the expressions of some randomly selected genes from different families were further confirmed by qRT-PCR (Additional file 2: Figure S8). Analysis of gene expression level (FPKM) showed that all these family genes have expressions in the root (Additional file 2: Figure S9A). Furthermore, results from our qRT-PCR analysis of selected DEGs from various families of auxin pathways in different tissues showed that these auxin-related genes were expressed in root, shoot and leaf, with different extent of expression in these tissues (Additional file 2: Figure S9B). In Arabidopsis, the YUC1, 2, 4 and 6 genes were downregulated in response to the exogenous NAA treatment through the feedback pathway [52]. In moso bamboo, 5 of the 13 PhYUC members responded to elevated auxin levels: PhYUC4, 5, 9 and 13 were significantly upregulated, and PhYUC3 was downregulated (Fig. 4, Additional file 4: Table S9).

In Arabidopsis, the expression of most auxin transporter genes are positively regulated by auxin, contributing to faster auxin transport when endogenous auxin is elevated [53]. In bamboo, we found that 3 PIN genes (PhPIN2, 4, 5) and 3 PILS genes (PhPILS1, 5, 8) changed their expression in response to exogenous auxin treatment. Auxin stimulated the expression of PhPIN2, 4, 5 and PhPILS1, 8, while downregulated the expression of PhPILS5 (Fig. 4, Additional file 5: Table S10).

In Arabidopsis roots, all $L A X$ genes are upregulated by auxin treatment [51]. However, we found that in moso bamboo, the expression of 2 PhLAX genes (PhLAX4 and $P h L A X 6)$ were downregulated by exogenous auxin treatment, whereas the transcriptional level of PhLAX3 was upregulated (Fig. 4, Additional file 6: Table S11).

For auxin signaling pathway, auxin receptor genes did not changed at transcriptional level (Additional file 7: Table S12). The most pronounced effects of the plant response to auxin are the upregulation of $A U X / I A A$ genes, and auxin signaling is activated by auxindependent degradation of proteins from this family [18]. In total, 21 PhIAA transcripts were upregulated by auxin in moso bamboo. Importantly, the transcription of PhIAA23, an ortholog of AtIAA19 in Arabidopsis and acts as the key regulator in Arabidopsis lateral root formation [54], was strongly induced in response to exogenous auxin. This result is quite similar to the data from Arabidopsis and other plants, indicating that auxin-activated $A U X / I A A$ gene upregulation is conserved in the plant kingdom (Fig. 4, Additional file 8: Table S13).

ARF proteins bind to the promoter elements and act as activators or repressors of downstream auxin responsive genes [21]. Of the 41 members of the ARF family identified in moso bamboo, 11 PhARFs were upregulated and 1 PhARF was downregulated by auxin (Fig. 4, Additional file 9: Table S14). Among the auxin responsive genes in Arabidopsis, AtARF19, an activator of auxin-dependent transcription is most sensitive to auxin at the transcriptional level. AtARF7 and AtARF19, which are phylogenetically close, are considered to be the only ARF factors that are necessary and sufficient for auxin signaling in 7-d-old light-grown seedlings [55]. Supporting this point, PhARF11, the ortholog of AtARF19, and PhARF13, the ortholog of $A t A R F 7$, were strongly induced by auxin (Fig. 4, Additional file 9: Table S14). AtARF2 acts as the communication node that links the ethylene and auxin signaling pathways [21], and its ortholog PhARF42 in bamboo was strongly induced by auxin. All the auxin pathway genes that changed expression at transcription level were listed in the Additional file 10: Table S15.

It is likely that moso bamboo possesses similar conserved signaling pathways that stimulated the downstream genes as other species, such as Arabidopsis. On the other hand, different mechanisms in controlling the auxin concentration exist between moso bamboo and Arabidopsis, for example, the expression of some auxin transporter genes in bamboo went down, whereas all their orthologs in Arabidopsis were upregulated after auxin treatment. Future analyses of the expression patterns and subcellular localizations, as well as the functional characterization of these genes will unveil the specific mechanisms of controlling the auxinconcentration related pathways in moso bamboo.

\section{Identification of additional important auxin-responsive transcription factors}

Except for the AUX/IAA family, 3 other gene families participate in the primary auxin response at the 


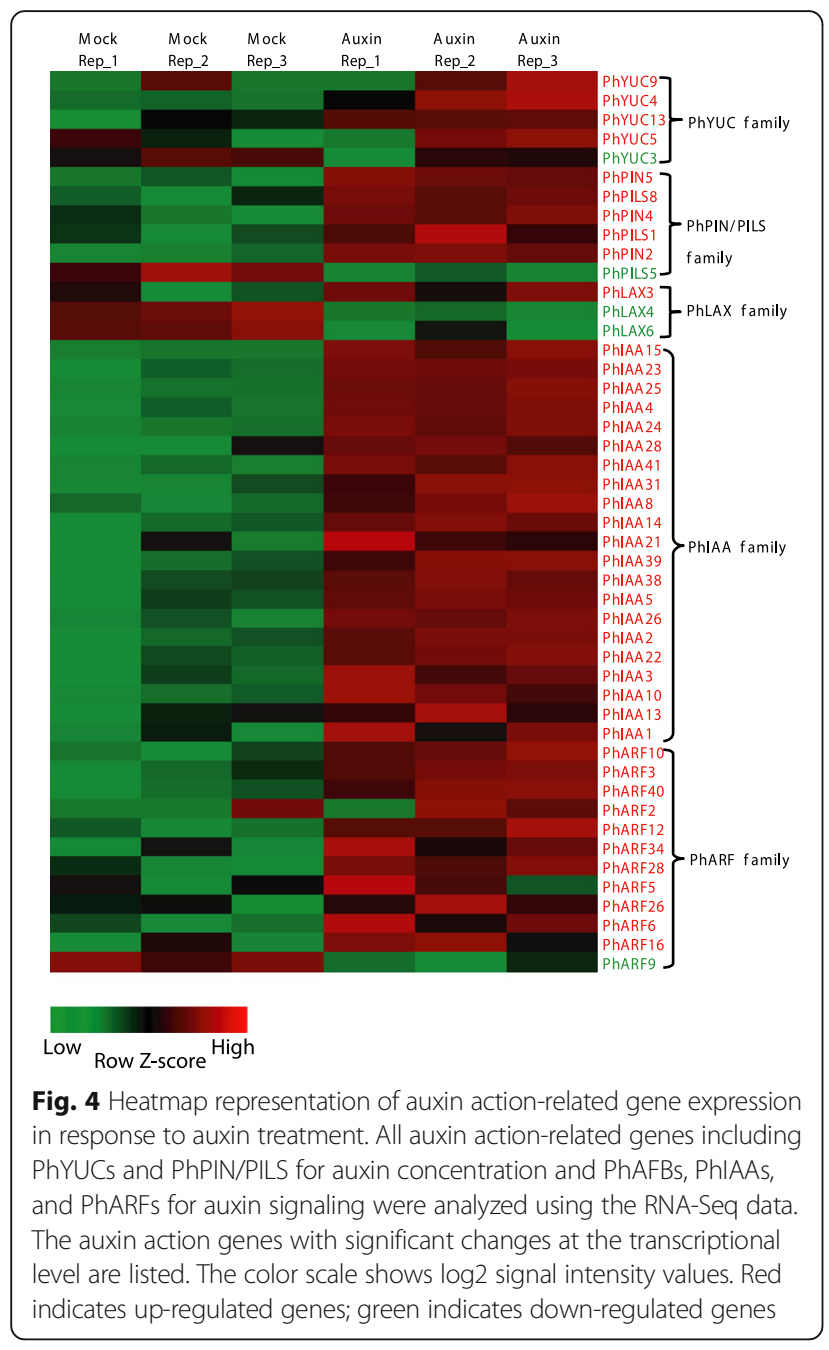

transcriptional level in Arabidopsis: Gretchen Hagen3 (GH3 family), small auxin-up RNA (SAUR family) and the lateral organ boundaries domain (LBD family) [51, $56,57]$. GH3 genes encode a class of auxin-induced conjugating enzymes and are auxin responsive genes involved in regulating auxin homeostasis and response [58]. At least 7 GH3 genes are strongly and rapidly induced by auxin in Arabidopsis [58]. Studies from Arabidopsis and the moss Physcomitrella patens suggested the evolutionarily conserved role for $G H 3$ proteins in regulating auxin homeostasis [59]. In the bamboo genome, we found 5 putative $G H 3$ genes with increased transcription in response to auxin (Additional file 11: Table S16). Among the early auxin response genes, the SAUR gene family has the largest number of member; it is estimated that around half of the Arabidopsis SAUR gene transcripts are rapidly upregulated by auxin, while a small number of this family members appear to be repressed by auxin [60,61]. Recently, a genome-wide analysis of SAUR genes was performed in moso bamboo [62]. Of the 38 SAUR genes identified, 10 SAUR genes changed their expressions under our auxin treatment conditions, the expression levels of 7 of those genes increased, whereas 3 genes decreased (Additional file 12: Table S17). The LATERAL ORGAN BOUNDARIES (LOB) proteins, which contain a conserved LOB domain (LBD), are rapidly and specifically up-regulated by auxin treatment [55]. Our RNA-Seq results also showed that in moso bamboo, at least 9 putative $L B D$ genes increased their transcripts in response to exogenous auxin treatment (Additional file 13: Table S18).

Based on the acid growth hypothesis, auxin stimulates protons pumping into the cell wall matrix, thereby helping to loosen the cell wall [63]. On the other hand, auxin may modulate cell wall properties by regulating the transcription of genes related to cell wall remodeling [64]. Consistent with this hypothesis, we found that a number of cell wall-related genes encoded structural cell wall proteins or enzymes changed their expressions after auxin treatment (Additional file 3: Table S8). In total, 51 genes, including expansins, xyloglucan endotransglycosylases (XTHs), and pectinmethylesterases (PMEs), were identified. Of these genes, 24 were upregulated and 27 were downregulated (Additional file 14: Table S19). Expansins were identified as the major cell wallloosening agents [63]. In bamboo, at least 5 expansinlike genes were upregulated at the transcriptional level (Additional file 14: Table S19). Xyloglucan endotransglycosylase /hydrolases that cut and paste xyloglucans, and endoglucanases which hydrolyze glucosidic bonds, also contribute to cell wall loosening and cell expansion by modifying cell wall properties and by integrating new wall materials, respectively [63]. Supporting to this point, 3 glucanase-related genes changed in expression after auxin treatment (Additional file 14: Table S19). Some glycanases that were transcriptionally increased by auxin catalyze the hydrolyses of cell wall polysaccharides, and are involved in the auxin-induced changes in the mechanical properties of cell walls [65]. We found that 4 genes encoding glucanases changed in expression after auxin treatment (Additional file 14: Table S19). Class III peroxidases induce cell wall loosening and growth by elongation as well as cross-linking of cell wall components. It should be noted here that the expression levels of two putative peroxidase genes were also changed (Additional file 14: Table S19). These results suggest that auxin induces a broad range of transcriptional changes in cell wall property-related genes.

\section{Crosstalk of auxin and other phytohormones in moso bamboo}

The crosstalk among hormone-regulated pathways was widely present in plant cells [37]. We further examined the interaction between auxin and other phytohormones. Cytokinin and auxin have long been recognized as 
crucial signaling molecules controlling plant growth and development [66]. Cytokinin is degraded by side chain cleavage through the action of the cytokinin oxidase/dehydrogenase (CKX) enzymes which are induced by auxin at the transcriptional level [67]. In moso bamboo, at least two putative cytokinin oxidases genes (PH01000072G1090 and PH01001279G0410) increased their transcriptional expression in the presence of auxin (Fig. 5a, Additional file 15: Table S20). Cytokinin conjugation is an important process that maintains the subcellular levels of active cytokinin. For example, the interactions between glycosides and cytokinins which are catalyzed by cytokinin-O-glucosylation inactivate cytokinins. We found that the expression of PH01021243G0010, a putative ortholog of cytokinin-O-glucosyltransferase 1 in maize, was upregulated. Type-A ARRs are negative regulators of cytokinin signaling in bamboo, one type-A ARR gene (PH01000999G0470) was upregulated by auxin (Fig. 5a, Additional file 15: Table S20). PH01000007G1150 is an ortholog of HAT22 which belongs to a family of cytokinin-regulated homeodomain zip (HD zip) class II transcription factors in Arabidopsis [68], and was activated by auxin at the transcriptional level in moso bamboo (Fig. 5a, Additional file 15: Table S20). Therefore auxin signaling pathways affect cytokinin synthesis, homeostasis, and other signaling pathways at the transcriptional level in moso bamboo as in other model plants like Arabidopsis and rice.

In total, 14 gibberellin acid (GA) related genes were responsive to auxin treatment in the moso bamboo root. Of these genes, 3 putative gibberellin-2-oxidases which are involved in GA degradation were downregulated. One putative Gibberellin-20-oxidase-2 which controls GA biosynthesis was upregulated (Fig. 5b, Additional file 16: Table S21). These results suggested that exogenous auxin controls GA levels through transcriptional regulation. A previous study in Arabidopsis showed that the effects of auxin on GA biosynthesis and signaling are complicated and depend on the concentration of exogenous auxin, duration of treatment or tissue type [51]. Therefore, more detailed analyses are needed to fully illustrate the crosstalk between auxin and GA.

The importance of auxin and ethylene crosstalk is wellestablished [69]. In total, 59 ethylene-related genes were found to be transcriptionally altered in response to auxin in the moso bamboo root (Fig. 5c, Additional file 17: Table S22). The application of auxin normally upregulates the transcription of genes encoding ethylene biosynthetic enzymes and leads to increased ethylene biosynthesis [69]. In accordance with this point, we found that 1 putative aminocyclopropane-1-carboxylic acid synthase $(A C S)$ gene (PH01000000G2120), which catalyzes the rate-limiting step in ethylene synthesis, was upregulated under our exogenous auxin treatment condition. The second group of auxin-responsive ethylene genes is involved in a signaling pathway. Twenty-five $E R F s$, including one putative ethylene receptor gene (PH01005673G0050), were auxin responsive, and most of these genes were upregulated (Fig. 5c, Additional file 17: Table S22). In general, the enhanced expression of ethylene biosynthesis and signaling genes after auxin treatment showed the positive crosstalk between these two plant hormones.

The auxin signaling pathway not only controls various aspects of plant growth and development but also plays roles in plant environmental adaptation via crosstalk with some stress-related phytohormones, such as abscisic acid (ABA), jasmonate (JA) or salicylic acid (SA) [37]. Our results showed that 20 genes involved in the ABA-related pathways are auxinresponsive (Fig. 5d, Additional file 18: Table S23). Of these, PH01000029G2070, the putative ABAresponsive element binding protein 3 (AREB3) transcription factor in moso bamboo, was strongly induced by exogenous auxin treatment. We further identified 32 and 30 auxin-responsive genes that are involved in the JA and SA pathways, respectively (Fig. 5e and f, Additional file 19: Table S24 and Additional file 20: Table S25).

\section{Discussion}

Bamboo is well known for its rapid growth and high level of woodiness. At the cellular level, the fast growth of the bamboo culm is mainly due to cell elongation and division [24]. Auxin is one of the well-known phytohormones that controls cell division and expansion [13]. However, the genes involved in auxin pathway and the global auxin-response profiling in moso bamboo still need to be investigated.

Here, we showed that exogenous NAA inhibit bamboo root growth (Fig. 1). We identified the key gene families involved in auxin biosynthesis (PhYUC family; 13 members), auxin transport (PhPIN, PhPILS and PhLAX families; 6, 8, and 7 members respectively), auxin receptors (PhAFB family; 10 members) and auxin signaling (PhIAA and $P h A R F$ families; 43 and 41 members, respectively) in moso bamboo (Fig. 2). Our analysis indicated the importance of compartmentalized auxin homeostasis and auxin signaling throughout the plant kingdom. Compared with Arabidopsis and rice, most of the auxin action- related gene families in moso bamboo have extensively expended their numbers of members (Additional file 2: Figure S4), suggesting that the auxin action in moso bamboo is more complicated and diverse.

Until now, no auxin-related gene in moso bamboo was functionally characterized. The phylogenetic relationships of the auxin pathway genes may suggest their putative 

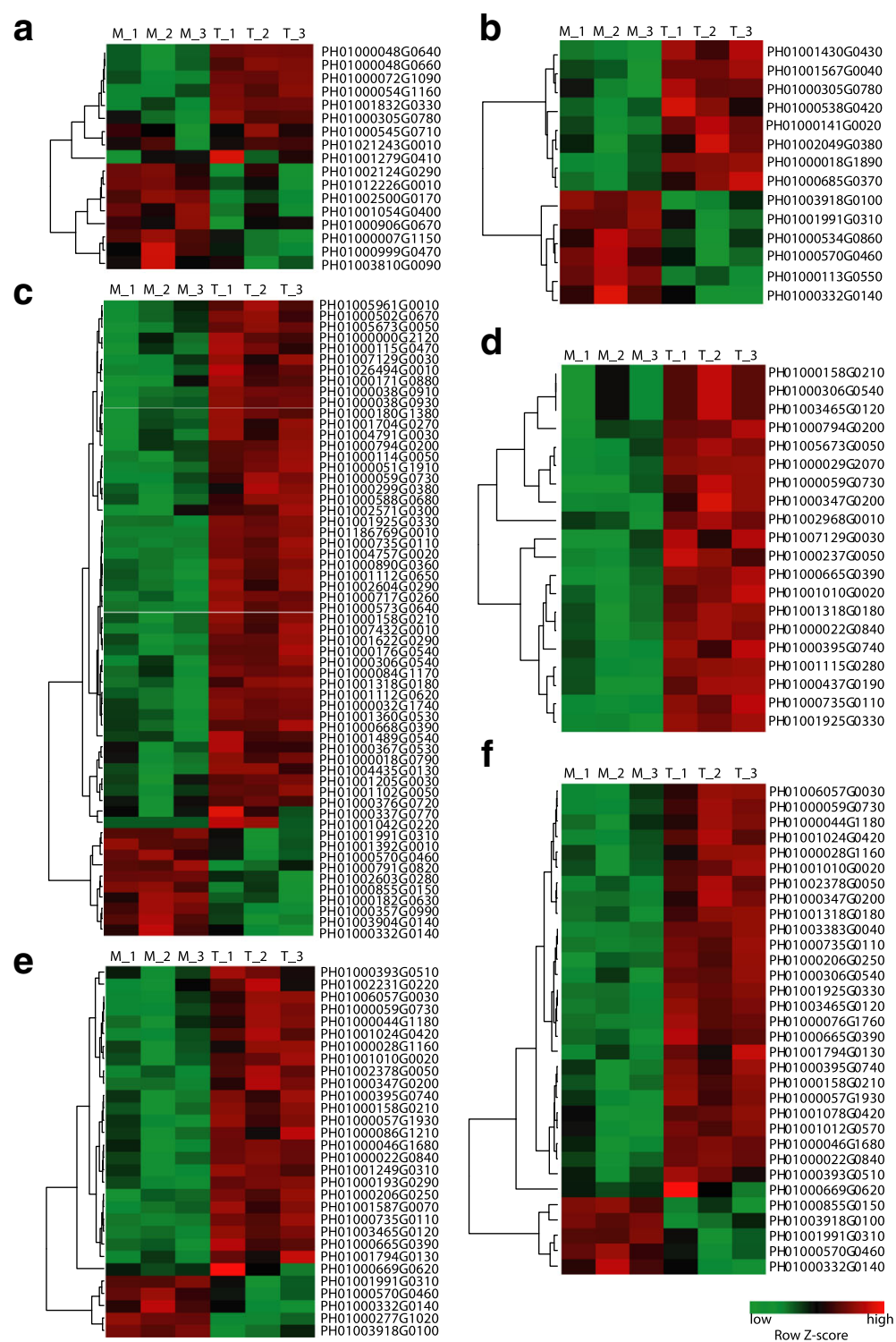

f

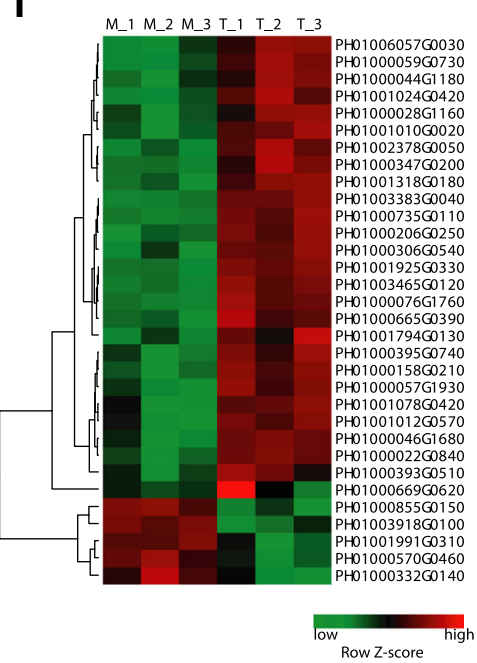

Fig. 5 Cross-talk between auxin and other phytohormones. The hierarchical clustering of auxin-responsive genes that are associated with cytokinin (a), ethylene (b), Gibberellin (c), ABA (d), SA (e), and JA (f) were shown. M_1-3, mocked sample with 3 repeats; A_1-3, auxin treated samples with 3 repeats. Color scale shows log2 signal intensity values. Red indicates up-regulated genes; green indicates down-regulated genes

roles in moso bamboo. For example, OsYUC1 encodes the key enzyme contributing to IAA biosynthesis in rice [70], and it will be very interesting to determine the function of its ortholog in moso bamboo PhYUC11 (Additional file 2: Figure S4). PhPIN4 is most closely related to OsPIN10a and OsPIN10b (Additional file 2: Figure S4), which are supposed to be monocot-specific [45]. In Arabidopsis, AtLAX3 is reported to promote the initiation of lateral root primordia by increasing the expression of a selection of cell-wall-remodeling enzymes [71]. In bamboo, we identified its closest ortholog PhLAX6 (Additional file 2: Figure S4). Heterodimerization between Aux/IAA and ARF proteins are crucial for their unique biological functions in different tissues in Arabidopsis [22]. The studies of the protein-protein interactions and gene coexpression maps in Arabidopsis, together with our phylogenetic analysis (Additional file 2: Figure S4), will facilitate future studies of the interaction maps of PhIAAs and PhARFs. This analysis is a key step to understanding the auxin action network in moso bamboo.

Previous studies have shown that elevated cellular auxin levels stimulate the transcriptional changes of genes involved in auxin biosynthesis, conjugation, transport, and signaling [51]. We performed the KEGG analysis of the differentially expressed genes, and our results showed that those differentially expressed genes were 
classified into 12 pathways, and the genes involved in plant hormone signal transduction were predominantly enriched (33 DEGs) (Additional file 21: Table S26). Our results also showed that a number of auxin pathway genes changed their expression, which is consistent with a previous report (Fig. 4, Additional file 10: Table S15). While there are some exceptions, for example, all $A U X 1 / L A X$ genes in Arabidopsis increased their transcripts after auxin treatment, but in moso bamboo, only 3 genes changed their expression levels: 2 PhLAX genes (PhLAX4 and PhLAX6) were down-regulated, whereas the YUC genes involved in auxin biosynthesis (PhYUC4, 5, 9 and 13) were mostly upregulated (Fig. 4). The latter result is opposite to the results in Arabidopsis, in which the $Y U C$ genes are downregulated through the feedback pathways [72]. These results indicate that the mechanisms that control auxin concentrations in moso bamboo are more complicated, and the expression of related genes may tightly and precisely controlled by the properties of external stimuli, different tissues, or various developmental stages. Interestingly, PhYUC3 is the only $Y U C$ gene that was downregulated by exogenous auxin in the moso bamboo root (Fig. 4), suggesting that PhYUC3 may encode one of the rate-limiting enzymes for auxin biosynthesis in the bamboo root. Further genetic and biochemical experiments are needed to characterize the potentially important function of these genes.

NAA, IAA and IBA are three most commonly used compounds in auxin research. Although they were transported in different ways in plant cells, they cause similar physiological response by changing overlapping downstream gene expressions [3, 73]. As a preliminary test on the effects of various auxins in moso bamboo, we treated the seedlings with $5 \mu \mathrm{M}$ IAA or $5 \mu \mathrm{M}$ IBA for $4 \mathrm{~h}$ (similar conditions as the NAA treatment we used), and randomly selected genes whose expressions were changed after NAA treatment for qRT-PCR analysis. The genes we checked include: Ph01003158G0110 and Ph01000099G0730 for GH3 family; Ph01000001G1450 and Ph01004534G0130 for SAUR family; Ph01001249G0310 for LBD family; Ph01000025G1600, Ph01000554G0550, Ph01003159G0070 and Ph01001154 G0590 for AUX/IAA family. Our results showed that these genes had similar expression patterns after IAA or IBA treatments, while the extents of the changes were different (Additional file 2: Figure S10), these results support the previous findings that different auxins have both common and specific efficacies to activate various auxin signaling pathways [74]. Moreover, previous reports also showed that different auxins cause distinct but overlapping changes in gene expression, probably due to the differences in their metabolism, transport or interaction with the signaling components [75, 76]. Thereby, it is needed to perform more detailed and systematic studies to unveil the effects of various auxins on the expression of auxin-related genes in moso bamboo in the future.

We also identified the genes that were regulated by exogenous auxin (Fig. 5). Notably, our results showed that a significant number of auxin-responsive genes are involved in the biosynthesis, metabolism or signaling pathway of other phytohormone like cytokinin, ethylene, Gibberellin, ABA, SA and JA (Fig. 5). Various plant hormones affect similar cellular processes through complicated interactions. Our results provide insights into how auxin pathways crosstalk with other plant hormone pathways to regulate moso bamboo growth and development, which should be investigated in the future.

\section{Conclusion}

In summary, we established a general overview of the main pathways involves in auxin synthesis, transport, receptor and signaling, and the global transcriptional profiling of auxin response in moso bamboo. The results from this study provided information for the elucidation of the possible functions of auxin actionrelated genes in bamboo. In the future, more experimental and bioinformatics work is needed to fully understand the functions of these important candidate genes and the regulatory mechanisms of some important auxin action-related proteins in this particular species.

\section{Additional files}

Additional file 1: Table S1. List of putative PhYUC family genes in maso bamboo. Table S2. List of putative PhPIN/PILS genes in maso bamboo. Table S3. List of putative PhLAX genes in maso bamboo. Table S4. List of putative auxin binding factors (AFB) in bamboo. Table S5. List of putative PhAUX/IAA genes in maso bamboo. Table S6. List of putative PhARF genes in maso bamboo. Table S7. List of primers used for qPCR analysis. (DOCX $28 \mathrm{~kb}$ )

Additional file 2: Figure S1. General overview of auxin biosynthesis, transport, and signaling pathway. Figure S2. Multiple sequence alignment, conserved motif and gene structure analysis of members involved in auxin concentration. Figure S3. Multiple sequence alignment, conserved motif and gene structure analysis of members involved in auxin signaling. Figure S4. Phylogenetic analysis of families related to auxin action in moso bamboo, rice, and Arabidopsis. Figure S5. qRT-PCR analysis of auxin responsive marker genes in response to exogenous auxin treatment. Figure S6. Heat-map of Euclidean distance among the 6 bamboo RNA-Seq libraries used in this study. Figure S7. Validation of RNA-Seq results by qPCR. Figure S8. qRTPCR confirmation of the expressions of auxin-related genes. Figure S9. Tissue expression patterns of the auxin related genes. Figure S10. qRT-PCR analysis of auxin responsive marker genes in response to exogenous IAA and IBA treatment. (PDF $3914 \mathrm{~kb}$ )

Additional file 3: Table S8. List of the genes in the RNA-Seq analysis. (TXT $4491 \mathrm{~kb}$ )

Additional file 4: Table S9. Expression of PhYUC family members in response to auxin treatment. (TXT $1 \mathrm{~kb}$ )

Additional file 5: Table S10. Expression of PhPIN/PhPILS family members in response to auxin treatment. (TXT $1 \mathrm{~kb}$ ) 
Additional file 6: Table S11. Expression of PhLAX family members in response to auxin treatment. (TXT $1 \mathrm{~kb}$ )

Additional file 7: Table S12. Expression of PhAFB family members in response to auxin treatment. (TXT $1 \mathrm{~kb}$ )

Additional file 8: Table S13. Expression of PhIAA family members in response to auxin treatment. (TXT $5 \mathrm{~kb}$ )

Additional file 9: Table S14. Expression of PhARF family members in response to auxin treatment. (TXT $5 \mathrm{~kb}$ )

Additional file 10: Table S15. List of the auxin action related genes with expression change in response to exogenous auxin treatment. (TXT $5 \mathrm{~kb}$ )

Additional file 11: Table S16. Expression of putative PhGH3 members in response to auxin treatment. (TXT 824 bytes)

Additional file 12: Table S17. Expression of PhSAUR family members in response to auxin treatment. (TXT $1 \mathrm{~kb}$ )

Additional file 13: Table S18. Expression of putative PhLBD members in response to auxin treatment. (TXT $1 \mathrm{~kb}$ )

Additional file 14: Table S19. Expression changes of cell wall related genes in response to exogenous auxin treatment. (TXT $6 \mathrm{~kb}$ )

Additional file 15: Table S20. Expression changes of cytokinin related genes in response to exogenous auxin treatment. (TXT $1 \mathrm{~kb}$ )

Additional file 16: Table S21. Expression changes of GA related genes in response to exogenous auxin treatment. (TXT $1 \mathrm{~kb}$ )

Additional file 17: Table S22. Expression changes of ethylene related genes in response to exogenous auxin treatment. (TXT $7 \mathrm{~kb}$ )

Additional file 18: Table S23. Expression changes of ABA related genes in response to exogenous auxin treatment. (TXT $2 \mathrm{~kb}$ )

Additional file 19: Table S24. Expression changes of JA related genes in response to exogenous auxin treatment. (TXT $3 \mathrm{~kb}$ )

Additional file 20: Table S25. Expression changes of SA related genes in response to exogenous auxin treatment. (TXT $3 \mathrm{~kb}$ )

Additional file 21: Table S26. The statistical enrichment of DEGs in the KEGG pathways. (TXT $4 \mathrm{~kb}$ )

\section{Abbreviations}

ABA: Abscisic acid; ACS: Aminocyclopropane-1-carboxylic acid synthase: AFB: Auxin signaling F-BOX protein; AREB: ABA-responsive element binding protein; ARFs: Auxin response factors; AUX/IAA: auxin/indole-3-aceticacid; AUX1/LAX: Auxin 1/like-AUX1; AuxRE: Auxin-responsive cis-element; BLAST: Basic local alignment search tool; CKX: Cytokinin oxidase/ dehydrogenase; CTD domain: Carboxy-terminal dimerization domain; DBD: DNA binding domain; DEGs: Differentially expressed genes; dUTP: Deoxyuridine triphosphate; ER: Endoplasmic reticulum; ERFs: Ethylene responsive factors; FDR: False discovery rate; FPKM: Fragment per kilobase of transcript per million mapped reads; GA: Gibberellin acid; GH3: Gretchen Hagen 3; HD zip: Homeodomain zip; HMM: Hidden Markov model; IAA: Indole-3-acetic acid; IBA: Indole-3-butytric acid; IPA: Indole-3-pyruvate; JA: Jasmonic acid; LBD: Lateral organ boundaries domain; LOB: Lateral organ boundaries; MS: Murashige and skoog; NAA: Naphthaleneacetic acid; ORF: Open reading frame; PIN/PILS: PIN-FORMED/PIN-like; PMEs: Pectinmethylesterases; qRT-PCR: Quantiative real time PCR; RNASeq: RNA-Sequencing; SA: Salicylic acid; SAUR: Small auxin-up RNA; SE: Standard error; TAA: Tryptophan aminotransferase; TIR1: Transport inhibitor response 1; XTHs: Xyloglucan endotransglycosylases; YUC: YUCCA

\section{Acknowledgements}

The authors thank the International Magnesium Institute for its financial support to W.J.W., and Prof. Yoshito Oka, Prof. Markus V. Kohnen, Prof. Liangsheng Zhang and Prof. Xu Chen for the critical reading of the manuscript.

\section{Funding}

This work was supported by Fujian Innovative Center for Germplasm Resources and Cultivation of Woody plants (no. 125/KLA15001E), and a grant from the Natural Science Foundation of Fujian province (no. 2016J01099) to Q.Z.. The National Natural Science Foundation of China grant (no. 31570674) to L.F.G. The funding bodies were not involved in the design of the study or in any aspect of the data collection, analysis and interpretation of data and in paper writing.

\section{Availability of data and materials}

RNA-Seq in this study had been submitted to GEO under accession number GSE100172.

\section{Authors' contributions}

QZ and LFG conceived this project; QZ, LFG and CTL designed experiments and interpreted the results. WJW and SWY performed the experiments, and HXZ, CYC, MQX, YBG and QW helped to collect and analyze the data. QZ wrote the manuscript. All authors read and approved the final manuscript.

Ethics approval and consent to participate

The plant materials used in this study were collected from Guangxi Zhuang Autonomous Region (Guangxi, China). This article did not contain any studies with human participants or animals performed by any of the authors. The experimental research on plants was conducted under the guidelines of the Convention on the Trade in Endangered Species of Wild Fauna and Flora.

Consent for publication

Not applicable.

\section{Competing interests}

The authors declare that they have no competing interests.

\section{Publisher's Note}

Springer Nature remains neutral with regard to jurisdictional claims in published maps and institutional affiliations.

\section{Author details}

${ }^{1}$ Basic Forestry and Proteomics Center (BFPC), Fujian Provincial Key Laboratory of Haixia Applied Plant Systems Biology, Haixia Institute of Science and Technology, Fujian Agriculture and Forestry University, Fujian 350002, China. ${ }^{2}$ Department of Molecular, Cell \& Developmental Biology, University of California, Los Angeles, California 90095, USA.

Received: 21 June 2017 Accepted: 31 October 2017

Published online: 13 November 2017

\section{References}

1. Balzan S, Johal GS, Carraro N. The role of auxin transporters in monocots development. Front Plant Sci. 2014;5:393.

2. Gallavotti A. The role of auxin in shaping shoot architecture. J Exp Bot. 2013; 64(9):2593-608.

3. Chapman EJ, Estelle M. Mechanism of auxin-regulated gene expression in plants. Annu Rev Genet. 2009;43:265-85.

4. Kasahara H. Current aspects of auxin biosynthesis in plants. Biosci Biotechnol Biochem. 2015;80(1):34-42.

5. Adamowski M, Friml J. PIN-dependent auxin transport: action, regulation, and evolution. Plant Cell. 2015;27(1):20-32.

6. Zhao Y. Auxin biosynthesis. Arabidopsis Book. 2014;12:e0173.

7. Mashiguchi K, Tanaka K, Sakai T, Sugawara S, Kawaide H, Natsume M, Hanada A, Yaeno T, Shirasu K, Yao H, et al. The main auxin biosynthesis pathway in Arabidopsis. Proc Natl Acad Sci U S A. 2011;108(45):18512-7.

8. Vieten A, Sauer M, Brewer PB, Friml J. Molecular and cellular aspects of auxin-transport-mediated development. Trends Plant Sci. 2007;12(4):160-8.

9. Grones P, Friml J. Auxin transporters and binding proteins at a glance. J Cell Sci. 2015:128(1):1-7.

10. Kramer EM, Bennett MJ. Auxin transport: a field in flux. Trends Plant Sci. 2006:11(8):382-6.

11. Barbez E, Kubes M, Rolcik J, Beziat C, Pencik A, Wang B, Rosquete MR, Zhu J, Dobrev $\mathrm{Pl}$, Lee $\mathrm{Y}$, et al. A novel putative auxin carrier family regulates intracellular auxin homeostasis in plants. Nature. 2012;485(7396):119-22.

12. Feraru E, Vosolsobe S, Feraru MI, Petrasek J, Kleine-Vehn J. Evolution and structural diversification of PILS putative Auxin carriers in plants. Front Plant Sci. 2012;3:227.

13. Benjamins R, Scheres B. Auxin: the looping star in plant development. Annu Rev Plant Biol. 2008;59(1):443-65. 
14. Dharmasiri N, Dharmasiri S, Weijers D, Lechner E, Yamada M, Hobbie L, Ehrismann JS, Jürgens G, Estelle M. Plant development is regulated by a family of Auxin receptor F box proteins. Dev Cell. 2005;9(1):109-19.

15. Dharmasiri N, Dharmasiri S, Estelle M. The F-box protein TIR1 is an auxin receptor. Nature. 2005;435(7041):441-5.

16. Yamada M, Greenham K, Prigge MJ, Jensen PJ, Estelle M. The TRANSPORT INHIBITOR RESPONSE2 gene is required for auxin synthesis and diverse aspects of plant development. Plant Physiol. 2009;151(1):168-79.

17. Mockaitis K, Estelle M. Auxin receptors and plant development: a new signaling paradigm. Annu Rev Cell Dev Biol. 2008;24:55-80.

18. Gray WM, Kepinski S, Rouse D, Leyser O, Estelle M. Auxin regulates SCF(TIR1)dependent degradation of AUX/IAA proteins. Nature. 2001;414(6861):271-6.

19. Szemenyei $H$, Hannon M, Long JA. TOPLESS mediates auxin-dependent transcriptional repression during Arabidopsis embryogenesis. Science. 2008; 319(5868):1384-6.

20. Hardtke CS, Ckurshumova W, Vidaurre DP, Singh SA, Stamatiou G, Tiwari SB, Hagen G, Guilfoyle TJ, Berleth T. Overlapping and non-redundant functions of the Arabidopsis auxin response factors MONOPTEROS and NONPHOTOTROPIC HYPOCOTYL 4. Development. 2004;131(5):1089-100.

21. Guilfoyle TJ, Hagen G. Auxin response factors. Curr Opin Plant Biol. 2007;10(5): 453-60.

22. Piya S, Shrestha SK, Binder B, Stewart CN Jr, Hewezi T. Protein-protein interaction and gene co-expression maps of ARFs and aux/IAAs in Arabidopsis. Front Plant Sci. 2014;5:744.

23. Buckingham KC, Wu L, Lou Y. Can't see the (bamboo) forest for the trees: examining bamboo's fit within international forestry institutions. Ambio. 2014;43(6):770-8.

24. Wang HY, Cui K, He CY, Zeng YF, Liao SX, Zhang JG. Endogenous hormonal equilibrium linked to bamboo culm development. Genet Mol Res. 2015; 14(3):11312-23.

25. Perrot-Rechenmann C. Cellular responses to auxin: division versus expansion. Cold Spring Harb Perspect Biol. 2010;2(5):a001446.

26. Peng Z, Lu Y, Li L, Zhao Q, Feng Q, Gao Z, Lu H, Hu T, Yao N, Liu K, et al. The draft genome of the fast-growing non-timber forest species moso bamboo (Phyllostachys Heterocycla). Nat Genet. 2013;45(4):456-61.

27. Larkin MA, Blackshields G, Brown NP, Chenna R, McGettigan PA, McWilliam H, Valentin F, Wallace IM, Wilm A, Lopez R, et al. Clustal W and Clustal X version 2.0. Bioinformatics. 2007;23(21):2947-8.

28. Skinner ME, Uzilov AV, Stein LD, Mungall CJ, Holmes $H_{H}$. JBrowse: a nextgeneration genome browser. Genome Res. 2009;19(9):1630-8.

29. Tamura K, Stecher G, Peterson D, Filipski A, Kumar S. MEGA6: molecular evolutionary genetics analysis version 6.0. Mol Biol Evol. 2013;30(12):2725-9.

30. Parkhomchuk D, Borodina T, Amstislavskiy V, Banaru M, Hallen L, Krobitsch S, Lehrach $\mathrm{H}$, Soldatov A. Transcriptome analysis by strandspecific sequencing of complementary DNA. Nucleic Acids Res. 2009; 37(18):20.

31. Kim D, Pertea G, Trapnell C, Pimentel H, Kelley R, Salzberg SL. TopHat2: accurate alignment of transcriptomes in the presence of insertions, deletions and gene fusions. Genome Biol. 2013;14(4):R36.

32. Trapnell C, Williams BA, Pertea G, Mortazavi A, Kwan G, van Baren MJ, Salzberg SL, Wold BJ, Pachter L. Transcript assembly and quantification by RNA-Seq reveals unannotated transcripts and isoform switching during cell differentiation. Nat Biotechnol. 2010; 28(5):511-5.

33. Robinson MD, McCarthy DJ, Smyth GK. edgeR: a bioconductor package for differential expression analysis of digital gene expression data. Bioinformatics. 2010;26(1):139-40.

34. Zhu Q, Dugardeyn J, Zhang C, Takenaka M, Kuhn K, Craddock C, Smalle J, Karampelias M, Denecke J, Peters J, et al. SLO2, a mitochondrial PPR protein affecting several RNA editing sites, is required for energy metabolism. Plant J. 2012;71(5):836-49.

35. Huang da W, Sherman BT, Tan Q, Kir J, Liu D, Bryant D, Guo Y, Stephens R, Baseler MW, Lane HC, et al. DAVID bioinformatics resources: expanded annotation database and novel algorithms to better extract biology from large gene lists. Nucleic Acids Res. 2007; 35(Web Server issue):W169-75.

36. Teale WD, Paponov IA, Palme K. Auxin in action: signalling, transport and the control of plant growth and development. Nat Rev Mol Cell Biol. 2006; 7(11):847-59.

37. Vanstraelen M, Benkova E. Hormonal interactions in the regulation of plan development. Annu Rev Cell Dev Biol. 2012;28:463-87.
38. De Smet I, Lau S, Voss U, Vanneste S, Benjamins R, Rademacher EH, Schlereth A, De Rybel B, Vassileva V, Grunewald W et al: Bimodular auxin response controls organogenesis in Arabidopsis. Proc Natl Acad Sci U S A 2010, 107(6):2705-2710.

39. Schlaich NL. Flavin-containing monooxygenases in plants: looking beyond detox. Trends Plant Sci. 2007:12(9):412-8.

40. Mohanta TK, Mohanta N, Bae H. Identification and expression analysis of PIN-like (PILS) gene family of Rice treated with Auxin and Cytokinin. Genes. 2015;6(3):622-40.

41. Yue R, Tie S, Sun T, Zhang L, Yang Y, Qi J, Yan S, Han X, Wang H, Shen C. Genome-wide identification and expression profiling analysis of ZmPIN, ZmPILS, ZmLAX and ZmABCB auxin transporter gene families in maize (Zea Mays L.) under various abiotic stresses. PLoS One. 2015;10(3):e0118751.

42. Swarup R, Peret B. AUX/LAX family of auxin influx carriers-an overview. Front Plant Sci. 2012:3:225

43. Kepinski S, Leyser $\mathrm{O}$. The Arabidopsis F-box protein TIR1 is an auxin receptor. Nature. 2005:435(7041):446-51.

44. Abu-Zaitoon YM, Bennett K, Normanly J, Nonhebel HM. A large increase in IAA during development of rice grains correlates with the expression of tryptophan aminotransferase OsTAR1 and a grain-specific YUCCA. Physiol Plant. 2012:146(4):487-99.

45. Wang JR, Hu H, Wang GH, Li J, Chen JY, Wu P. Expression of PIN genes in rice (Oryza Sativa L.): tissue specificity and regulation by hormones. Mol Plant. 2009;2(4):823-31.

46. Xu H-W, Y-w M, Wang W, Wang H, Wang Z. OsPIN1a gene participates in regulating negative phototropism of Rice roots. Rice Sci. 2014;21(2):83-9.

47. Paul P, Dhandapani V, Rameneni JJ, Li X, Sivanandhan G, Choi SR, Pang W, Im S, Lim YP. Genome-wide analysis and characterization of aux/IAA family genes in Brassica Rapa. PLoS One. 2016;11(4):e0151522.

48. Singh VK, Jain M. Genome-wide survey and comprehensive expression profiling of aux/IAA gene family in chickpea and soybean. Front Plant Sci. 2015;6:918.

49. Song Y, Wang L, Xiong L. Comprehensive expression profiling analysis of OsIAA gene family in developmental processes and in response to phytohormone and stress treatments. Planta. 2009;229(3):577-91.

50. Zhu ZX, Liu Y, Liu SJ, Mao CZ, Wu YR, Wu P. A gain-of-function mutation in OsIAA11 affects lateral root development in rice. Mol Plant. 2012;5(1):154-61.

51. Paponov IA, Paponov M, Teale W, Menges M, Chakrabortee S, Murray JA, Palme K. Comprehensive transcriptome analysis of auxin responses in Arabidopsis. Mol Plant. 2008;1(2):321-37.

52. Suzuki M, Yamazaki C, Mitsui M, Kakei Y, Mitani Y, Nakamura A, Ishii T, Soeno K, Shimada Y. Transcriptional feedback regulation of YUCCA genes in response to auxin levels in Arabidopsis. Plant Cell Rep. 2015; 34(8):1343-52.

53. Zažímalová E, Murphy AS, Yang H, Hoyerová K, Hošek P. Auxin transporters-why so many? Cold Spring Harb Perspect Biol. 2010;2(3): a001552.

54. Tatematsu K, Kumagai S, Muto H, Sato A, Watahiki MK, Harper RM, Liscum E, Yamamoto KT. MASSUGU2 encodes auX/IAA19, an auxin-regulated protein that functions together with the transcriptional activator NPH4/ARF7 to regulate differential growth responses of hypocotyl and formation of lateral roots in Arabidopsis Thaliana. Plant Cell. 2004;16(2):379-93.

55. Okushima Y, Overvoorde PJ, Arima K, Alonso JM, Chan A, Chang C, Ecker JR, Hughes B, Lui A, Nguyen D, et al. Functional genomic analysis of the AUXIN RESPONSE FACTOR gene family members in Arabidopsis Thaliana: unique and overlapping functions of ARF7 and ARF19. Plant Cell. 2005;17(2):444-63.

56. Okushima Y, Fukaki H, Onoda M, Theologis A, Tasaka M. ARF7 and ARF19 regulate lateral root formation via direct activation of $L B D / A S L$ genes in Arabidopsis. Plant Cell. 2007;19(1):118-30.

57. Hagen G, Guilfoyle T. Auxin-responsive gene expression: genes, promoters and regulatory factors. Plant Mol Biol. 2002;49(3-4):373-85.

58. Staswick PE, Serban B, Rowe M, Tiryaki I, Maldonado MT, Maldonado MC, Suza W. Characterization of an Arabidopsis enzyme family that conjugates amino acids to indole-3-acetic acid. Plant Cell. 2005;17(2):616-27.

59. Ludwig-Muller J, Julke S, Bierfreund NM, Decker EL, Reski R. Moss (Physcomitrella Patens) GH3 proteins act in auxin homeostasis. New Phytol. 2009:181(2):323-38

60. Jain M, Tyagi AK, Khurana JP. Genome-wide analysis, evolutionary expansion, and expression of early auxin-responsive SAUR gene family in rice (Oryza Sativa). Genomics. 2006;88(3):360-71. 
61. Ren H, Gray WM. SAUR proteins as effectors of hormonal and environmental signals in plant growth. Mol Plant. 2015;8(8):1153-64

62. Bai Q, Hou D, Li L, Cheng Z, Ge W, Liu J, Li X, Mu S, Gao J. Genome-wide analysis and expression characteristics of small auxin-up RNA (SAUR) genes in moso bamboo (Phyllostachys Edulis). Genome. 2016;15(10):2016-0097.

63. Cosgrove DJ. Growth of the plant cell wall. Nat Rev Mol Cell Biol. 2005;6(11):850-61.

64. Lewis DR, Olex AL, Lundy SR, Turkett WH, Fetrow JS, Muday GK. A kinetic analysis of the Auxin Transcriptome reveals Cell Wall remodeling proteins that modulate lateral root development in Arabidopsis. Plant Cell. 2013; 25(9):3329-46.

65. Kotake T, Nakagawa N, Takeda K, Sakurai N. Auxin-induced elongation growth and expressions of cell wall-bound exo- and endo-beta-glucanases in barley coleoptiles. Plant Cell Physiol. 2000;41(11):1272-8.

66. Zdarska M, Dobisova T, Gelova Z, Pernisova M, Dabravolski S, Hejatko J. Illuminating light, cytokinin, and ethylene signalling crosstalk in plant development. J Exp Bot. 2015;66(16):4913-31.

67. Schmulling T, Werner T, Riefler M, Krupkova E, Bartrina y Manns I. Structure and function of cytokinin oxidase/dehydrogenase genes of maize, rice, Arabidopsis and other species. J Plant Res. 2003;116(3):241-52.

68. Brenner WG, Romanov GA, Kollmer I, Burkle L, Schmulling T: Immediateearly and delayed cytokinin response genes of Arabidopsis thaliana identified by genome-wide expression profiling reveal novel cytokininsensitive processes and suggest cytokinin action through transcriptional cascades. Plant J 2005, 44(2):314-333.

69. Muday GK, Rahman A, Binder BM. Auxin and ethylene: collaborators or competitors? Trends Plant Sci. 2012;17(4):181-95.

70. Yamamoto Y, Kamiya N, Morinaka Y, Matsuoka M, Sazuka T. Auxin biosynthesis by the YUCCA genes in rice. Plant Physiol. 2007;143(3):1362-71.

71. Swarup K, Benkova E, Swarup R, Casimiro I, Peret B, Yang Y, Parry G, Nielsen E, De Smet I, Vanneste $S$, et al. The auxin influx carrier LAX3 promotes lateral root emergence. Nat Cell Biol. 2008;10(8):946-54.

72. Zhao Y, Dai X, Blackwell HE, Schreiber SL, Chory J. SIR1, an upstream component in auxin signaling identified by chemical genetics. Science. 2003;301(5636):1107-10.

73. Vanneste S, Friml J. Auxin: a trigger for change in plant development. Cell. 2009:136(6):1005-16.

74. Simon S, Kubes M, Baster P, Robert S, Dobrev PI, Friml J, Petrasek J, Zazimalova E. Defining the selectivity of processes along the auxin response chain: a study using auxin analogues. New Phytol. 2013;200(4):1034-48.

75. Pufky J, Qiu Y, Rao MV, Hurban P, Jones AM. The auxin-induced transcriptome for etiolated Arabidopsis seedlings using a structure/function approach. Funct Integr Genomics. 2003;3(4):135-43.

76. Woodward AW, Bartel B. Auxin: regulation, action, and interaction. Ann Bot. 2005;95(5):707-35.

\section{Submit your next manuscript to BioMed Central and we will help you at every step:}

- We accept pre-submission inquiries

- Our selector tool helps you to find the most relevant journal

- We provide round the clock customer support

- Convenient online submission

- Thorough peer review

- Inclusion in PubMed and all major indexing services

- Maximum visibility for your research

Submit your manuscript at wuw biomedcentral.com/submit

) Biomed Central 